\title{
QUALIDADE NUTRICIONAL E CONTAMINANTES DE ALFACE (Lactuca sativa L.) CONVENCIONAL, ORGÂNICA E HIDROPÔNICA
}

\section{NUTRITIONAL QUALITY AND CONTAMINANTS OF LETTUCE (Lactuca sativa L.) FROM CONVENTIONAL, ORGANIC AND HYDROPONIC SYSTEM}

\author{
STERTZ, S.C. ${ }^{1}$; FREITAS, R.J.S. ${ }^{2}$; ROSA, M.I.S ${ }^{3}$; PENTEADO, P.T.P.S. ${ }^{4}$ \\ RECEBIDO: JUNHO 2005 ACEITE: AGOSTO 2005
}

1.Doutora em Tecnologia de Alimentos - Programa de Pós-graduação em Tecnologia de Alimentos UFPR - Química, Pesquisadora, Setor de Tecnologia, Departamento de Engenharia Química, Universidade Federal do Paraná - Caixa Postal 19011 CEP 81531-990 Curitiba PR - Brasil. E-mail: stertz@ufpr.br.

2.Doutor em Engenharia Química DEQ/UPR -Professor do Programa de Pós-Graduação em Tecnologia de Alimentos DEQ-UFPR.

3.Química, Pesquisadora, Setor de Tecnologia, Laboratório de Química Analítica Aplicada - UFPR

4.Doutora em Meio Ambiente e Desenvolvimento, UFPR Professora da Disciplina de Tecnologia de Alimentos Dietéticos do curso de Farmácia - UFPR.

\begin{abstract}
RESUMO
Foram analisadas amostras de alface oriundas dos sistemas de cultivo convencional, orgânico e hidropônico na Região Metropolitana de Curitiba - RMC, em relação à sua composição nutricional e contaminantes. Foram observadas algumas tendências, sendo que as alfaces hidropônicas apresentaram maiores teores de $\mathrm{Mg}$, $\mathrm{Na}$, Co e $\mathrm{Cu}$ e as alfaces orgânicas apresentaram menores teores de nitritos $(46,7 \%)$ e de nitratos $(157,4 \%)$ quando comparadas às convencionais. Enquanto as alfaces orgânicas apresentaram-se isentas de resíduos de agrotóxicos, as convencionais e as hidropônicas apresentaram, respectivamente, $33,33 \%$ e $66,67 \%$ de suas amostras contaminadas.
\end{abstract}

Palavras-chave: Lactuca sativa L., controle de qualidade; agrotóxicos; minerais; nitritos e nitratos.

\begin{abstract}
The lettuce samples were analyzed from conventional, organic and hydroponic systems in the Metropolitan Area of Curitiba - MAC, regarding the nutritional composition and contaminants. Some tendencies were observed, the hydroponic lettuces showed higher contents of $\mathrm{Mg}, \mathrm{Na}$, Co and $\mathrm{Cu}$ and the organic lettuces showed lower contents of nitrites $(46.7 \%)$ and nitrates $(157.4 \%)$ when compared in relation to the conventional system. While the organic lettuces came exempt of agrotoxics residues, conventional ones and hydroponics presented, respectively, $33.33 \%$ and $66.67 \%$.
\end{abstract}

Keywords: Lactuca sativa L., quality control; agrotoxics; nitrites and nitrates

\section{INTRODUÇÃO}

Dentre as diversas alternativas alimentares, destacam-se as hortaliças folhosas, entre elas a alface (Lactuca sativa L.), uma das mais presentes à mesa do consumidor, sendo responsável por cerca de 4,5\% (48.450 toneladas) de toda a produção de hortaliças no Paraná (PARANÁ, 2004).

A procura por alimentos orgânicos é expressiva em todo o mundo, devido a conscientização da população sobre os riscos, para a saúde, decorrentes da presença de resíduos químicos nos alimentos (MACHADO e CORAZZA, 2004). O mercado mundial de produtos orgânicos cresceu na década de 90 , cerca de $10 \%$ ao ano, mas na virada deste milênio houve um aceleração vertiginosa, com estimativas de crescimento 
em torno de 40 a $50 \%$, em termos de volume de produtos comercializados (CHAIM, 2002). Estima-se que a área cultivada sob manejo orgânico no Brasil seja de aproximadamente 275 mil hectares, sendo cerca de 158 mil com agricultura e 119 mil de pastagens (YUSSEFI e WILLER, 2003). A produção orgânica no Paraná destaca-se nacionalmente, com crescimento superior a $1200 \%$ nas ultimas 8 safras, sendo que as principais culturas exploradas são a cana-de-açúcar, soja, hortícolas e frutas. A agricultura da Região Metropolitana de Curitiba (RMC) está voltada basicamente para a produção de hortícolas, respondendo por $50 \%$ da produção do Estado do Paraná (HAMERSCHMIDT, 2005).

Enquanto estudos que comparam os alimentos obtidos pelos sistemas de cultivo convencional e orgânico, em relação ao seu valor nutricional, têm sido realizados, há poucos capazes de conclusões válidas cientificamente, com exceção do conteúdo de nitrato, matéria seca, vitamina $\mathrm{C}$ e alguns antioxidantes (BOURN e PRESCOT, 2002; AFSSA, 2003). Esses dados evidenciam a necessidade de novos e criteriosos estudos.

Nesse sentido, o objetivo geral deste trabalho foi avaliar a qualidade da alface convencional, orgânica e hidropônica na RMC, em relação às características nutricionais e contaminantes.

\section{MATERIALE MÉTODOS}

\subsection{COLETAE PREPARO DAAMOSTRA}

Em dezembro de 2001, foram coletadas, de acordo com os procedimentos para coleta de amostras (BRASIL, 2001; CCE, 2001) 18 amostras de alface crespa, cv. Verônica (Lactuca sativa L., Compositaceae), no estádio de maturação comercial e disponíveis para consumo; junto ao CEASA-PR, propriedades rurais e associações (AOPA, APAC) da RMC. Esta cultivar está inserida no grupo das alfaces que apresentam folhas soltas, crespas, consistentes, sem formação de cabeça (FILGUEIRA, 2003).

Os lotes das amostras, com cerca de 5 quilos cada, foram coletados, acondicionados em sacos de polietileno, transportados em caixas de isopor com gelo, devidamente rotulados, até o laboratório de Química Analítica Aplicada/UFPR, em um período máximo de três horas após a coleta.

As amostras foram ligeiramente lavadas com água corrente e água destilada (para eliminar sujidades), secas com guardanapo de papel (para retirar o excesso de umidade).

As determinações de $\mathrm{pH}$ e umidade foram feitas com as amostras in natura.

Para a determinação de resíduos de agrotóxicos, as amostras foram trituradas em processador de alimentos em baixa rotação (3.000 rpm) por dois minutos, congeladas em potes de plástico com boca larga, fechados com tampas também de plástico, e armazenadas em congelador $\mathrm{a}-18^{\circ} \mathrm{C} \pm 2^{\circ} \mathrm{C}$ até o momento da análise. Os reagentes foram grau pesticida (Mallinckrodt) e padrões (Fluka), utilizando-se processador de alimentos (Wallita Mega Master Pro), balão com três bocas esmerilhadas com $500 \mathrm{ml}$ de capacidade, equipamento para evolução do $\mathrm{CS}_{2}$ com duas armadilhas, banho ultratermostático a $15^{\circ} \mathrm{C}$ (Tecnal mod TE-184), espectrofotômetro (Milton Roy - mod. Spectronic Genesys 5), HPLC (marca HP, modelo HP 1100 com 
detector de UV), CG Massav (marca HP, modelo HP 5890 com detector de ECD e NPD).

Para as demais determinações, as amostras foram levadas à estufa com circulação forçada de ar (Marconi MA 035), na temperatura de $55-60^{\circ} \mathrm{C}$, durante 12 horas. Após homogeneizadas e trituradas em um multiprocessador de alimentos, em baixa rotação (3000 rpm) por dois minutos, foram passadas em tamis de $2 \mathrm{~mm}$ de $\varnothing$ e acondicionadas em potes plásticos com tampa até o momento da análise.

\subsection{MÉTODOS UTILIZADOS}

Composição centesimal As proteínas foram determinadas pelo nitrogênio total, utilizando o método de Kjeldahl (macro-Kjeldahl Prodicil) e o fator de 6,25 para conversão em proteína (AOAC, 2000 - 955.04C). O extrato etéreo (lipídios) foi determinado por extração com éter etílico durante cinco horas em extrator de Soxhlet (Prodicil) (AOAC, 2000 - 920.39C). As cinzas foram determinadas pela calcinação em mufla (Quimis) a $550-600^{\circ} \mathrm{C}$ durante cinco horas (AOAC, 2000 - 900.02A). A umidade foi determinada em estufa (Marconi MA 035) com circulação forçada de ar, com temperatura de $55-60^{\circ} \mathrm{C}$ durante 12 horas, ou até peso constante (AOAC, 2000 925.10). Os carboidratos totais foram calculados por diferença: (100 g - total g (proteína, lipídios, cinzas), portanto já inclui a fração fibra alimentar (USP, 2001).

A energia total metabolizável foi calculada a partir da energia procedente dos nutrientes, considerando os fatores de conversão de Atwater: $\mathrm{kcal}=(4 \times \mathrm{g}$ proteína $)+(4 \times \mathrm{g}$ carboidratos (carboidratos totais - fibra alimentar $))+(9 \times \mathrm{g}$ lipídios) (USP, 2001).

Os sólidos totais (matéria seca) foram calculados pela diferença entre 100 e a umidade.

O pH foi determinado por potenciometria (Peagâmetro Orion, modelo $710^{\mathrm{A}}$ ) (AOAC, 2000 -981.12).

A fibra alimentar foi determinada utilizando-se uma combinação de métodos enzimático e gravimétrico (AOAC, 2000 - 992.16). Foram utilizadas as enzimas $\alpha$ amilase (Termamyl 120 L, Novo Nordisk A/S), protease (Savinase 16 L, Novo Nordisk A/S) e amiloglugosidase (AMG 300 L, Novo Nordisk A/S) e Banho-maria Dubnoff (Nova Ética, mod. 304).

Os açúcares glucose, sacarose, frutose e maltose foram determinados por cromatografia líquida de alta eficiência - CLAE (Controlador Waters 600 E Millipore; Waters 712 WISP Millipore; Detector de Índice de Refreção 1037 A HP) (AOAC, 2000 982.14). Foram utilizados padrões da Sigma-Aldrich para a frutose (d(-)Fructose, 0,05

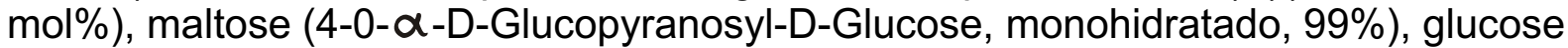
(D-(+)Glucose, Dextrose, 99,5\%) e sacarose ( $\alpha$-D- Glucopyranosyl $\beta$ - D-frutofuranoside, $>99,5 \%)$.

Em relação aos minerais, foram determinados os teores de alumínio, cálcio, cádmio, chumbo, cobalto, cobre, ferro, fósforo, magnésio, manganês, mercúrio, potássio, sódio, selênio e zinco (AOAC, 2000 - 999.10). As amostras foram homogeneizadas e $400 \mathrm{mg}$ foram digeridas com $\mathrm{HNO}_{3}$ em um forno de microondas (DGT 100, Provecto Sistemas Analíticos) por 15 minutos. A solução foi diluída com água bi-deionizada até uma concentração adequada para cada elemento e transferida para frasco de polietileno. Procedeu-se a leitura através de uma Fonte Geradora de Plasma Acoplado a um Espectrômetro de Massa - ICP-MS (PE SCIEX ELAN 6000; Nebulizador do tipo "cross-flow", com "Ryton Spray Chamber"; Autosampler Perkin Elmer AS 91 
acoplado a um FIAS 400), com o auxílio de uma curva padrão para cada elemento.

Os teores de nitritos e nitratos foram determinados conforme metodologia $4110 \mathrm{~B}$, descrita no STANDARD METHODS (1998). Pesou-se 0,5g de amostra seca, em balão volumétrico de $100 \mathrm{ml}$ com água destilada e deionizada, agitando-se ocasionalmente por uma hora. Completou-se o volume e filtrou-se em papel filtro faixa preta (Whatmann 389). Procedeu-se a leitura direta em um Cromatógrafo lônico de Alta Eficiência (HPIC), Dionex modelo DX-500, consistindo de coluna lon-Pac AS14A (4×250 mm); pré-coluna AG-14 (4x50 mm); detetor de condutividade modelo ED 40; amostrador automático modelo AS 40, observando-se as recomendações do manual do usuário da DIONEX (1999), bem como os parâmetros de calibração e validação da metodologia utilizada. As condições de operação foram: eluição isocrática; fluxo 1,2 $\mathrm{mL} / \mathrm{minuto}$; volume de injeção $50 \mu \mathrm{L}$; limite de deteção $0,05 \mathrm{mg} / \mathrm{L}$; limite de quantificação $0,05 \mathrm{mg} / \mathrm{L}$; eluente mistura de $\mathrm{Na}_{2} \mathrm{CO}_{3}(8 \mathrm{mmol} / \mathrm{L})$ e $\mathrm{NaHCO}_{3}(1 \mathrm{mmol} / \mathrm{L})$.

Para a análise de resíduos de agrotóxicos nas amostras, os teores de multiresíduos foram quantificados por varredura em cromatografia gasosa, para os grupos químicos organoclorados, organofosforados, carbamatos e piretróides, sendo pesquisadas as moléculas Aldrin, Alfa-BHC, Beta-BHC, Bifemox, Clorotalonil, Deltametrina, Dieldrin, Endossulfan I, Endossulfan II, Endossulfan sulfato, Endrin, Endrin aldeído, Fenarimol, Heptacloro, Heptacloro epóxido, Imidan, Iprodione, Lindane, Metolaclor, Metoxicloro, o,p'Diclofol, p,p'Dicofol, Propiconazole, Trifuralina, Vinclozolin, 4,4'-DDD, 4,4'-DDE, 4,4'-DDT, Clorfenvinfós, Clorpirifós, Cumafós, Demeton, Diazinon, Diclorvós, Dimetoato, Dissulfoton, Etion, Etoprofós, Fenclorfós, Fenitrotion, Fention, Forato, Fosfamidon, Metamidofós, Metidation, Mevinfós, Naled, Paration metílico, Pirazofós, Pirimifós metílico, Tocution, Triazofós, Tricloronato, Carbofuran, Carbaril, Methiocarb, Permetrina e Monocrotofós (CDFA, 1999a). Também foram determinados os teores de ditiocarbamatos (KEPPEL, 1971), por quantificação do dissulfeto de carbono $\left(\mathrm{CS}_{2}\right)$ por espectrofotometria e benzimidazóis, para os princípios ativos Carbendazim (methyl benzimidazol -2- ylcarbamate), Benomil [1- (butylcarbamoyl) benzimidazol -2- ylcarbamate)] e Tiofanato metílico [dimethyl 4,4' - (o-phenylene) bis (3thioallophanato)], por cromatografia líquida (CDFA, 1999b).

\subsection{ANÁLISE ESTATÍSTICA}

Os dados obtidos foram analisados estatisticamente pelo programa MSOFFICE MICROSOFT EXCEL (MICROSOFT, 1997) para o cálculo das médias, desvio padrão e coeficiente de variância. Com o auxílio do programa MINITAB (1998), os dados foram submetidos à análise de variância e teste $\mathrm{F}$ e Teste de Tukey para verificar a ocorrência de diferenças estatisticamente significativas $(p \leq 0,05)$ entre os cultivos.

\section{RESULTADOS E DISCUSSÃO}

De um modo geral, os dados encontrados no presente trabalho apresentaram grande variação entre os valores mínimo e máximo, o que pode justificar os altos valores no desvio padrão. Em alguns casos, o desvio padrão excede a média, fato que explica a não ocorrência de diferença estatística significativa entre algumas das culturas analisadas, quando comparadas em relação ao sistema de cultivo. 
As amostras de alface analisadas, em relação às cinzas e magnésio, não apresentaram diferenças estatísticas significativas $(p \leq 0,05)$, quando comparados os sistemas de cultivo orgânico e convencional, diferindo, porém, das amostras obtidas pelo sistema de cultivo hidropônico (Tabela 1). Considerando-se os minerais analisados, as amostras apresentaram algumas tendências positivas quando comparado o sistema de cultivo hidropônico em relação ao convencional, com cerca de $+38 \%$ de $\mathrm{Mg},+72 \%$ de $\mathrm{Na},+2000 \%$ de $\mathrm{Co}$ e $+78 \%$ de $\mathrm{Cu}$.

TABELA 1 - DETERMINAÇÕES FÍSICO-QUÍMICAS EM AMOSTRAS DE ALFACE OBTIDAS PELOS SISTEMAS DE CULTIVO CONVENCIONAL, ORGÂNICO E HIDROPÔNICO RMC

\begin{tabular}{|c|c|c|c|c|}
\hline Determinações & Unidade & $\begin{array}{c}\text { Convencional } \\
\mathrm{n}^{(1)}=6 \\
\text { Média } \pm \mathrm{DP}(2)\end{array}$ & $\begin{array}{c}\text { Orgânico } \\
\mathrm{n}^{(1)}=6 \\
\text { Média } \pm \mathrm{DP}(2)\end{array}$ & $\begin{array}{l}\text { Hidropônico } \\
\mathrm{n}^{(1)}=6 \\
\text { Média } \pm \mathrm{DP}(2)\end{array}$ \\
\hline Umidade & $\mathrm{g}$ & $94,18 \pm 0,57^{n s}$ & $94,46 \pm 0,49^{\text {ns }}$ & $94,77 \pm 1,01^{\text {ns }}$ \\
\hline Matéria seca & $\mathrm{g}$ & $5,83 \pm 0,57^{n s}$ & $5,54 \pm 0,49^{n s}$ & $5,23 \pm 1,01^{\text {ns }}$ \\
\hline Cinzas * & $\mathrm{g}$ & $0,94 \pm 0,10^{a}$ & $0,95 \pm 0,18^{a}$ & $0,74 \pm 0,16^{b}$ \\
\hline Fibras & g & $1,97 \pm 0,11^{\mathrm{ns}}$ & $1,67 \pm 0,19^{\text {ns }}$ & $1,65 \pm 0,50^{\text {ns }}$ \\
\hline Lipídios & $\mathrm{g}$ & $0,27 \pm 0,03^{\text {ns }}$ & $0,29 \pm 0,04^{\mathrm{ns}}$ & $0,29 \pm 0,08^{\text {ns }}$ \\
\hline Proteínas & $\mathrm{g}$ & $1,21 \pm 0,25^{\text {ns }}$ & $1,10 \pm 0,12^{n s}$ & $1,33 \pm 0,27^{n s}$ \\
\hline Carboidratos & $\mathrm{g}$ & $3,39 \pm 0,46^{n s}$ & $3,22 \pm 0,47^{n s}$ & $2,87 \pm 0,89^{n s}$ \\
\hline Energia & kcal & $13 \pm 2,32^{\mathrm{ns}}$ & $13 \pm 1,16^{\text {ns }}$ & $13 \pm 2,73^{\text {ns }}$ \\
\hline $\mathrm{pH}$ & & $6,12 \pm 0,26^{\text {ns }}$ & $6,15 \pm 0,37^{\mathrm{ns}}$ & $6,05 \pm 0,38^{\text {ns }}$ \\
\hline Frutose & g & $0,26 \pm 0,07^{n s}$ & $0,24 \pm 0,07^{n s}$ & $0,30 \pm 0,07^{n s}$ \\
\hline Glucose & $\mathrm{g}$ & $0,12 \pm 0,07^{n s}$ & $0,13 \pm 0,06^{\mathrm{ns}}$ & $0,13 \pm 0,05^{\text {ns }}$ \\
\hline Maltose & g & $0,00 \pm 0,00^{\mathrm{ns}}$ & $0,00 \pm 0,00^{\text {ns }}$ & $0,09 \pm 0,21^{\text {ns }}$ \\
\hline Sacarose & $\mathrm{g}$ & $0,14 \pm 0,07^{n s}$ & $0,12 \pm 0,05^{\mathrm{ns}}$ & $0,22 \pm 0,16^{n s}$ \\
\hline Soma dos acúcares & $\mathrm{g}$ & $0,53 \pm 0,13^{\text {ns }}$ & $0,49 \pm 0,16^{\mathrm{ns}}$ & $0,73 \pm 0,43^{\text {ns }}$ \\
\hline \multicolumn{5}{|l|}{ Minerais } \\
\hline Cálcio, Ca & $\mathrm{mg}$ & $17,58 \pm 4,14^{\mathrm{ns}}$ & $17,59 \pm 3,72^{\text {ns }}$ & $18,71 \pm 9,94^{\text {ns }}$ \\
\hline Fósforo, $P$ & $\mathrm{mg}$ & $16,23 \pm 6,67^{\mathrm{ns}}$ & $14,66 \pm 4,14^{\text {ns }}$ & $16,45 \pm 5,73^{\text {ns }}$ \\
\hline Magnésio, Mg & $\mathrm{mg}$ & $13,37 \pm 5,24^{b}$ & $8,79 \pm 7,26^{b}$ & $18,40 \pm 5,10^{a}$ \\
\hline Potássio, $\mathrm{K}^{\star \star}$ & $\mathrm{mg}$ & $258,92 \pm 46,57^{a}$ & $159,45 \pm 56,3^{b}$ & $187,16 \pm 88,82^{b}$ \\
\hline Sódio, $\mathrm{Na}$ & $\mathrm{mg}$ & $3,05 \pm 2,98^{\mathrm{ns}}$ & $1,06 \pm 0,92^{\mathrm{ns}}$ & $5,23 \pm 6,57^{\mathrm{ns}}$ \\
\hline Cobalto, Co & $\mu \mathrm{g}$ & $0,34 \pm 0,18^{\text {ns }}$ & $0,26 \pm 0,09^{n s}$ & $7,11 \pm 16,03^{\text {ns }}$ \\
\hline Cobre, Cu & $\mathrm{mg}$ & $0,10 \pm 0,14^{\mathrm{ns}}$ & $0,08 \pm 0,12^{\text {ns }}$ & $0,17 \pm 0,29$ ns \\
\hline Ferro, Fe & $\mathrm{mg}$ & $1,21 \pm 1,04^{\mathrm{ns}}$ & $0,59 \pm 0,26^{\mathrm{ns}}$ & $0,87 \pm 0,85^{\text {ns }}$ \\
\hline Manganês, Mn & $\mathrm{mg}$ & $0,24 \pm 0,18^{\mathrm{ns}}$ & $0,13 \pm 0,08^{\mathrm{ns}}$ & $0,21 \pm 0,16^{\mathrm{ns}}$ \\
\hline Selênio, Se & $\mu \mathrm{g}$ & $3,77 \pm 1,47^{n s}$ & $3,49 \pm 2,33^{\text {ns }}$ & $1,79 \pm 0,77^{n s}$ \\
\hline Zinco, Zn & $\mathrm{mg}$ & $0,23 \pm 0,09^{n s}$ & $0,14 \pm 0,03^{n s}$ & $0,21 \pm 0,12^{\text {ns }}$ \\
\hline \multicolumn{5}{|l|}{ Metais pesados } \\
\hline Alumínio, $\mathrm{Al}$ & $\mathrm{mg}$ & $0,80 \pm 0,78^{n s}$ & $0,33 \pm 0,22^{n s}$ & $0,26 \pm 0,37^{n s}$ \\
\hline Cádmio, Cd & $\mu g$ & $0,89 \pm 0,90 \mathrm{~ns}$ & $0,64 \pm 0,35^{n s}$ & $0,39 \pm 0,46^{n s}$ \\
\hline Mercúrio, $\mathrm{Hg}$ & $\mu \mathrm{g}$ & $0,69 \pm 0,97 \mathrm{~ns}$ & $0,20 \pm 0,12^{n s}$ & $0,19 \pm 0,20^{\text {ns }}$ \\
\hline Chumbo, $\mathrm{Pb}$ & $\mu \mathrm{g}$ & $2,77 \pm 1,25^{n s}$ & $3,73 \pm 2,21^{\mathrm{ns}}$ & $2,61 \pm 0,75^{\text {ns }}$ \\
\hline
\end{tabular}

NOTA: : a, b - médias na mesma linha com letras iguais não diferem estatisticamente no nível de $5 \%$ $(p \leq 0,05) ; \quad$ ns - não significativo. * $p=0,055 ;{ }^{* *} p=0,053 ;$ Os resultados correspondem à média das amostras, analisadas em triplicata; (1) $n$ = número de amostras analisadas; (2) Valores por $100 \mathrm{~g}$ de amostra, expressos em base úmida.

PFEILSTICKER (1992) obteve níveis mais altos de minerais (K, Mg, Fe, Mn, Cd e Zn) nas amostras de alfaces cultivadas pelo sistema biodinâmico, quando comparadas 
com o convencional, compatíveis parcialmente ( $\mathrm{Mg}$, Fe e $\mathrm{Cd})$ com os dados obtidos no presente estudo (Tabela 1).

Na Tabela 2 observa-se teores de nitrato na faixa de $264 \mathrm{mg} / \mathrm{kg}, 430 \mathrm{mg} / \mathrm{kg}$ e $1108 \mathrm{mg} / \mathrm{kg}$ para as amostras de alface orgânica, convencional e hidropônica, respectivamente, ou seja, $\mathrm{O}<\mathrm{C}<\mathrm{H}$. Esses resultados estão concordantes com dados apresentados por BOURN e PRESCOT (2002) e AFSSA (2003) e também com estudos de MIYAZAWA et al. (2001), que obtiveram essa mesma relação (base seca) quando analisaram 101 amostras de alface convencional, orgânica e hidropônica.

TABELA 2 - TEORES DE NITRITOS $\left(\mathrm{NO}_{2}^{-}\right)$E NITRATOS $\left(\mathrm{NO}_{3}^{-}\right)$EM AMOSTRAS DE ALFACE OBTIDAS PELOS SISTEMAS DE CULTIVO CONVENCIONAL, ORGÂNICO E HIDROPÔNICONARMC

\begin{tabular}{|c|c|c|c|c|c|c|}
\hline Determinações ${ }^{*}$ & \multicolumn{2}{|c|}{ Convencional } & \multicolumn{2}{|r|}{ Orgânico } & \multicolumn{2}{|r|}{ Hidropônico } \\
\hline & $\mathrm{n}$ & Média $\pm \mathrm{DP}$ & $\mathrm{n}$ & Média $\pm \mathrm{DP}$ & $\mathrm{n}$ & Média \\
\hline $\mathrm{NO}_{2}^{-}$ & & $368,53 \pm 449,63^{\text {ns }}$ & & $196,14 \pm 334,36^{\text {ns }}$ & & $670,94 \pm 424,87^{\text {ns }}$ \\
\hline $\mathrm{NO}_{3}^{-}$ & & $430,34 \pm 386,59^{n s}$ & & $263,83 \pm 162,66^{\text {ns }}$ & & $1107,70 \pm 1424,96^{n s}$ \\
\hline $\mathrm{S}$ & 06 & $798,86 \pm 679,78^{a}$ & 06 & $459,97 \pm 347,61^{a}$ & 06 & $1778,64 \pm 1308,61^{b}$ \\
\hline
\end{tabular}

NOTA: $\mathrm{S}=$ soma dos teores de nitritos e nitratos; $\mathrm{n}=$ número de amostras analisadas; $\mathrm{DP}=$ desvio padrão; *valores apresentados em base úmida, em $\mathrm{mg} / \mathrm{kg} ; \mathrm{a}, \mathrm{b}$ - médias na mesma linha com letras iguais não diferem estatisticamente entre si no nível de $5 \%(p \leq 0,05)$; ns - não significativo.

As amostras de alface convencional e hidropônica foram as que apresentaram as maiores restrições ao consumo, variando de cerca de 330 e $148 \mathrm{~g}$, considerando a IDA para nitritos e nitratos fixada pela Comissão Européia (EC, 1992 e 1998) e FAO/WHO (1996), para uma pessoa com $70 \mathrm{~kg}$.

Para a AFSSA (2003), os métodos de agricultura biológica/orgânica permitem a redução de 30 a $50 \%$ nos teores de nitratos das hortícolas, em relação aos métodos convencionais, sendo que os métodos de fertilização (essencialmente orgânicos) autorizados para esse tipo de cultura (BRASIL, 1999, 2002b, 2003) explicam as diferenças observadas. Essa redução parece interessante, uma vez que a ingestão diária total de nitratos na França é pouco inferior à dose diária aceitável e que as recomendações nutricionais atuais apontam para um aumento no consumo de hortícolas, devido a seus efeitos potencialmente protetores (doenças cardiovasculares, câncer), ricos em fibras, vitaminas e outros micronutrientes.

Das 18 amostras de alface analisadas, 33,33\% apresentaram algum tipo de resíduo de agrotóxicos (Tabela 3 ), sendo os mais presentes o manebe e o mancozebe, fungicidas comumente utilizados em pulverização de olerícolas. 33,33\% das amostras de alface convencional e $66,67 \%$ das hidropônicas apresentaram presença de mancozebe em concentrações acima do LMR permitido pela legislação brasileira (BRASIL, 2002a e 2004), configurando uma irregularidade e um risco à saúde do consumidor. Tais resultados são compatíveis com os últimos monitoramentos de resíduos de agrotóxicos em hortaliças no Estado do Paraná (PARANÁ, 2003), onde 11 das 37 amostras de alface convencional analisadas apresentaram resíduos de agrotóxicos, o que representa $30 \%$ do total de amostras. 
TABELA 3 - RESÍDUOS DE AGROTÓXICOS EM AMOSTRAS DE ALFACE ORGÂNICAS, CONVENCIONAIS E HIDROPÔNICAS, EM RELAÇÃO AOS PRINCÍPIOS ATIVOS DETECTADOS, NARMC 2001-2002

\begin{tabular}{|c|c|c|c|c|c|}
\hline $\begin{array}{l}\text { Sistema de } \\
\text { cultivo }\end{array}$ & $\begin{array}{l}\mathrm{N}^{\circ} \text { de mostras } \\
\text { c/resíduos }\end{array}$ & $\begin{array}{l}\mathrm{N}^{\circ} \text { de } P A_{(1)}^{(1)} \\
\text { Detectado }\end{array}$ & $\begin{array}{c}\text { Concentração } \\
(\mathrm{mg} / \mathrm{kg})\end{array}$ & $\begin{array}{l}\text { LMR (2) } \\
(\mathrm{mg} / \mathrm{kg})\end{array}$ & $\mathrm{UNP}^{(3)}$ \\
\hline \multirow[t]{5}{*}{ Convencional } & $2(33,33 \%)$ & 4 & 2,03 & & \\
\hline & & Manebe & 2,10 & 10,00 & \\
\hline & Am. 1 & Propinebe & $2,29^{(4)}$ & 1,00 & \\
\hline & & Manebe & 1,78 & 10,00 & \\
\hline & Am. 2 & Propinebe & $1,95^{(4)}$ & 1,00 & \\
\hline Orgânico & $0(0,00 \%)$ & 0 & 0,00 & & \\
\hline \multirow[t]{10}{*}{ Hidropônico } & $4(66,67 \%)$ & 9 & 2,36 & & \\
\hline & & Manebe & 2,63 & 10,00 & \\
\hline & Am. 3 & Propinebe & $2,88^{(4)}$ & 1,00 & \\
\hline & & Clorotalonil & $0,04^{(4)}$ & & $\mathbf{X}$ \\
\hline & Am. 4 & Manebe & 1,62 & 10,00 & \\
\hline & & Propinebe & $\mathbf{1 , 7 7 ^ { ( 4 ) }}$ & 1,00 & \\
\hline & & Manebe & 4,94 & 10,00 & \\
\hline & Am. 5 & Propinebe & $\mathbf{5 , 3 9} 9^{(4)}$ & 1,00 & \\
\hline & & Manebe & 0,96 & 10,00 & \\
\hline & Am. 6 & Propinebe & $\mathbf{1 , 0 5 ^ { ( 4 ) }}$ & 1,00 & \\
\hline TOTAL & $6(33,33 \%)$ & 13 & 2,10 & & \\
\hline
\end{tabular}

NOTA: $N^{\circ}$ total de amostras analisadas: 18 (cada amostra corresponde a uma propriedade diferente); ${ }^{(1)}$ $\mathrm{PA}=$ Princípio Ativo (ou molécula); ${ }^{(2)} \mathrm{LMR}=$ Limite Máximo de Resíduos; ${ }^{\left({ }^{3}\right)}$ UNP= Uso Não Permitido; $\mathbf{X}=$ Presença de princípio ativo de uso não permitido (UNP) para a respectiva cultura; ${ }^{(4)}$

Todas as amostras de alface orgânica apresentaram-se isentas de qualquer tipo de resíduo de agrotóxico (Tabela 3). Segundo CALDAS e SOUZA (2000), a garantia de alimento livre de contaminantes é essencial para a prevenção de doenças, principalmente em um país como o Brasil, onde uma parte considerável da população enfrenta sérios problemas de carência nutricional e de acesso ao sistema publico de saúde.

O clorotalonil é um fungicida organoclorado, cujo ingrediente ativo apresentavase na época em reavaliação técnica pela ANVISA (RDC no 135 de 17/05/02). Ele não é autorizado para a cultura analisada há muitos anos, no entanto, no presente estudo (Tabela 3) foi detectado resíduo deste princípio ativo (UNP) em uma amostra de alface hidropônica.

\section{CONCLUSÕES}

Apesar de terem sido observadas poucas diferenças significativas em relação às características nutricionais, a alface orgânica apresentou menores teores de nitrito e nitrato e ausência de qualquer tipo de resíduo de agrotóxico, tendo, portanto, um maior apelo em relação à segurança alimentar e à qualidade de vida.

As amostras de alface hidropônica apresentaram níveis mais altos, maior 
freqüência, mais resíduos múltiplos, com maiores riscos associados ao consumo, configurado pela desinformação do consumidor, uma vez que o mesmo acredita ser esse sistema de cultivo livre de agrotóxicos

\section{REFERÊNCIAS}

AFSSA. Evaluation nutritionnelle et sanitaire dês aliments issus de lágriculture biologique. Disponível em: <http://www.afssa.fr.htm>. Acesso em: 10 out. 2003.

AOAC - Association of Official Analytical Chemists. Official methods of analysis of AOAC International. 17ed., Gaithersburg, 2000. v.1-2.

BOURN, D.; PRESCOTT, J. A comparison of the nutritional value, sensory qualities, and food safety of organically and a conventionally produced foods. Critical Reviews in Food Science and Nutrition, Dunedin, New Zealand, v.42, n.1, p.1-34, 2002.

BRASIL. Normas disciplinadoras para a produção, tipificação, processamento, envase, distribuição, identificação e certificação da qualidade de produtos orgânicos, sejam de origem animal ou vegetal. IN nº 007 de 17 de maio de 1999, DOU de 19 de maio de 1999.

Ministério da Saúde. Agência Nacional de Vigilância Sanitária, Procedimentos de Retirada de Amostras, ANEXO $\mathrm{V}$ da Agência Nacional de Vigilância Sanitária. Disponível em: <http://www.anvisa.gov.br/alimentos/tox/manual/anexo 05.htm>Acesso em: 02 fev 2001.

Ministério da Saúde. Agência Nacional de Vigilância Sanitária. Monografias de produtos agrotóxicos. Resolução RE n.165, de 29 de agosto de 2003. D.O.U de 02/09/ 2003. Disponível em: <www.anvisa.gov.br/alimentos/tox/mono/index.htm>Acesso em: 2 jan. 2002a e 13 jan. 2004.

Secretaria de Defesa Agropecuária. Instrução Normativa $n^{\circ} 6$, de 10 de Janeiro de 2002. Dispõe sobre termos credenciamento, inspeção e certificação de Produção Orgânica. Diário Oficial da União da República Federativa do Brasil, 16 de janeiro de 2002b.

Presidência da República. Lei n 10.831, de 23 de dezembro de 2003. Dispõe sobre termos credenciamento, inspeção e certificação de Produção Orgânica. Diário Oficial da União da República Federativa do Brasil, 24 de dezembro de 2003, seção 1, página 8. 2003.

CALDAS, E. D.; SOUZA, L. C. K. Avaliação de risco crônico da ingestão de resíduos de pesticidas na dieta brasileira. Revista de Saúde Publica, v.34, n.5, p.529-37, 2000.

CCE. Comissão das Comunidades Européias. Recomendação da Comissão, de 3 de março de 1999. Jornal Oficial $n^{\circ}$ L 128 de 21/05/1999. p. 0025-0055. Disponível em: <http://europa.eu.int/eurlex/pt/lif/dat/1999/pt_399HO333.html> Acesso em 22 fev. 2001.

CDFA. California Department of Food and Agriculture. Multiresidue screen for pesticides in fruits and vegetables. 3.ed. Sacramento, CA: FDA, 1999a.

CDFA. California Department of Food and Agriculture. Benomyl, Carbendazin and Thiophanatemethyl Analysis. 3.ed. Sacramento, CA: FDA, 1999b.

DIONEX Corporation. AS14AManual. Doc. No. 031678-01, 34 p. Sunnyvale, CA, 1999.

EC. European Commission, Scientific Committee for Food, Report of the Scientific Committee for Food on Nitrate and Nitrite (26 ${ }^{\text {th }}$ series). [Opinion expressed on 19 October 1990], 1992.

EC. European Commission, Reports of the Scientific Committee for Food ( $38^{\text {th }}$ series). Opinion on Nitrates and Nitrites, Luxembourg: Office for Official Publications of the European Communities), p.1-33 , 1998.

FILGUEIRA, F.A.R. Novo Manual de Olericultura: agrotecnologia moderna na produção e comercialização de hortaliças. 2.ed Viçosa: UFV, 20, 412p

HAMERSCHMIDT, I. Panorama geral: os números da agricultura orgânica hoje destacando o Paraná. Disponível em: <http://www.planetaorganico.com.br/trabiniberto.htm>. Acesso em 20 de abril de 2005.

JECFA FAO/WHO. Joint Expert Committee on Food Additives FAO/WHO. Toxicological evaluation of certain food additives and contaminants in food. WHO Food Additives Series 35, Geneva, WHO, 1996.

KEPPEL, G. E. Collaborative study of the determination of dithiocarbamate residues by a modified carbon disulfide evolution method. Journal of the Association of Official Analytical Chemists, Washington, v.54, n.3. p.528-532, 1971.

MACHADO, F.; CORAZZA, R. Desafios tecnológicos, organizacionais e financeiros da agricultura orgânica no Brasil. Revista de la Facultad de Ecominía, v.26, p.21-40, 2004. 
Microsoft Corporation. MSoffice Microsoft Excel 97. Redmond, WA, c 1995-1997. CDROM 6 MB.

MINITAB. Reference Manual Release 7. 13 Demo. Statistical Software 13.0. Minitab Inc, 3081, Enterprise Drive, State Collegey PA, 16801 US, Elinor Cruze and Jim Weldon. 6-10, 6-11, 1998.

MIYAZAWA, M.; KHATOUNIAN, C.A.; ODENATH-PENHA, L.A. Teor de nitrato nas folhas de alface produzida em cultivo convencional, orgânico e hidropônico. Agroecologia Hoje. 9: 23, 2001.

PARANÁ. Secretaria de Estado da Saúde. Relatório do Programa de Análise de Resíduos de Agrotóxicos em Alimentos no Estado do Paraná, junho de 2001 a junho de 2002/ Secretaria de Estado da Saúde. - Curitiba: SESA, 2003.55p.

PARANÁ. SEAB/DERAL. Hortaliças Paraná Evolução da Área Colhida e da Produção Obtida 03/04. Disponível em: <http//www.pr.gov.br/seab/deral/ehpr.xls>. Acesso em: 11 de out 2004.

PFEILSTICKER, K., Quality of organic-grown food an experimental, multifactorial approach on vegetables for example. In: INTERNATIONAL IFOAM SCIENTIFIC CONFERENCE, $9^{\text {th }}$, Proceedings...1992, Brasil. Germany: Ifoam, 1992, p. 331-337.

STANDARD METHODS for the Examination of Water and Wastewater. 20 ed. American Public Health Association, Washington, 1998.

USP. Universidade de São Paulo. Tabela brasileira de composição de alimento: projeto integrado de composição de alimentos. Disponível em: http://www.fcf.usp.br/tabela/tbcacoce.php. Acesso em: 10 dez. 2001.

YUSSEFI, M.; WILLER, $\mathrm{H}$. The world of organic agriculture: statistcs and future prospects. Disponível em: <http://www.ifoam.org >. Acesso de 05 de dezembro de 2003.

AGRADECIMENTOS: Os autores agradecem o apoio financeiro fornecido pela Fundação Araucária-PR Projeto 567. 